\title{
Prescribing errors involving antineoplastics and others drug centre of preparation of injectable drugs
}

\author{
Luciana dos SANTOS, Thalita JACOBY, Sandro NESS, Gérson GUERRA, Carlos Alberto WAYHS \\ ${ }^{1}$ Hospital de Clínicas de Porto Alegre \\ Corresponding author: Santos LD, lusantos@hcpa.edu.br \\ Submitted: 02-08-2018 Resubmitted: 27-12-2019 Accepted: 28-12-2019 \\ Peer review: blind reviewers
}

\begin{abstract}
Objective: To describe the prescribing errors involving antineoplastics and others drugs in a centre for the preparation of injectable drugs at a university hospital. Method: A retrospective descriptive study was carried out based on the records of a drug preparation center with prescribing errors identified in the pharmaceutical validation phase prior to drug preparation in the period from 2016 to 2017.Results: A total of 1516 prescriptions/month were evaluated and 562 prescribing errors were identified and the prescription error rate involving medications was 1.5\%. Of the drugs most involved in errors are cisplatin (37.5\%), etoposide (14.1\%), carboplatin (8.9\%), cyclophosphamide (5.7\%) and oxaliplatin (4.1\%). Most of the errors were related to the diluents associated with the preparations, either in the absence of this information or in the prescription of volumes outside the concentration range required by the preparation of the drug with $56 \%$ and $22.6 \%$ respectively. In $94.3 \%$ of the prescriptions identified with errors, pharmaceutical interventions were necessary for its correction before preparation with adhesion in $99.6 \%$ of the cases. Conclusion: Although prescribing errors are described in the literature, the study presents the fragility of the prescriber system, even when it is computerized, and the importance of organized barriers or processes to avoid errors of prescription and manipulation in a centre for the preparation of injectable drugs.
\end{abstract}

Keywords: medication errors, drug prescriptions, antineoplastic agents.

\section{Erros de prescrição envolvendo quimioterápicos e outros medicamentos numa central de preparos de injetáveis}

\begin{abstract}
Resumo
Objetivo: Descrever os erros de prescrição envolvendo quimioterápicos e outros medicamentos numa central de preparo de medicamentos injetáveis de hospital universitário. Método: Realizou-se estudo descritivo retrospectivo com base nas informações de uma central de preparo de medicamentos injetáveis sobre erros de prescrição identificados na etapa de validação farmacêutica antes do preparo do medicamento no período de 2016 a 2017. Resultados: Foram avaliadas em média 1516 prescrições/mês e identificaram-se 562 erros de prescrição e a taxa de erros de prescrição envolvendo medicamentos foi de 1,5\%. Dentre os medicamentos mais envolvidos em erros estão cisplatina (37,5\%), etoposido (14,1\%), carboplatina (8,9\%), ciclofosfamida $(5,7 \%)$ e oxaliplatina $(4,1 \%)$. A maioria dos erros esteve relacionada com os diluentes associados aos preparos, seja na falta desta informação ou na prescrição de volumes fora da faixa de concentração exigida pelo preparo do medicamento com $56 \%$ e 22,6\%, respectivamente. Em 94,3\% das prescrições identificadas com erros foram necessárias intervenções farmacêuticas para sua correção antes do preparo com adesão em 99,6\% dos casos. Conclusão: Apesar dos erros de prescrição estarem bem descritos na literatura, o estudo mostra a fragilidade do sistema prescritor, mesmo sendo informatizado, e a importância de barreiras ou processos organizados para se evitar erros de prescrição e de manipulação numa central de preparos de medicamentos injetáveis.
\end{abstract}

Palavras-chave: erros de medicação, prescrição de medicamentos, agentes antineoplásicos.

\section{Introduction}

Errors related to medication use are among the most common causes of morbidity and mortality in patients ${ }^{1}$. A medication error is any preventable event that may cause the inappropriate use of medications, even without causing harm².
In a study carried out by the World Health Organization in 58 Latin American hospitals, it was identified that, out of 11 thousand hospitalized patients, approximately $10 \%$ were exposed to some type of harm during hospitalization and, considering the increase in hospital stay, the risk of exposure to some harmful event has doubled to $20 \%^{3}$. The occurrence of harms to patients, or drug- 
related morbidity, can reach $7.1 \%$ in outpatients and $6.5 \%$ in inpatients. In outpatients, $58.9 \%$ of this morbidity could have been avoided and, in hospital patients, harms could have been avoided in up to $41 \%$ of the cases ${ }^{4}$.

Chemotherapy drugs are at high risk for errors with serious consequences ${ }^{5}$. These drugs are involved in $15.4 \%$ of the errors with fatal outcome ${ }^{6}$. These errors are due to several factors, among them, the narrow therapeutic index that many drugs have; the toxic effects that can occur even during treatment with regular doses; the high number of therapeutic protocols, as well as the extensive supportive therapy associated, usually involving several drugs with different dosage schedules ${ }^{7}$. Many strategies are employed in an attempt to reduce the occurrence of errors in the use of chemotherapy, such as training of the professionals responsible for handling chemotherapy, elaboration of standard procedures for correct and safe handling of medications, and analysis of treatment protocols, among others ${ }^{8}$. Through these actions, the pharmacist plays an important role in ensuring the safe and rational use of these drugs ${ }^{6}$.

Among all the measures taken to ensure safety in the medication use process, the analysis of prescriptions is one of the most significant. The pharmacist must evaluate all components present in the prescription, such as quantity, compatibility, quality, stability, interactions and, also, examine the protocols established by the multidisciplinary team of antineoplastic therapy ${ }^{9}$. Prescription of incorrect doses, omission of some medication in an involuntary way or incorrect name of the drug, differences in the protocol cycle to be followed, incorrect administration route, and inadequate infusion time are some examples of errors ${ }^{10}$

According to the Institute for Safe Practices in the Use of Medications, chemotherapy drugs are considered potentially dangerous medications, as they have an increased risk for causing significant medication errors to the patients; the type of error (prescription and administration, among others) will define the complexity and consequence of the effects or harms to the patient, such as vincristine, where errors with serious outcomes are reported. Due to these factors, the ISMP and other organizations that work with patient safety recommend that the health teams that work with these drugs know their risks and create strategies to implement practices that help to minimize the occurrence of errors in health institutions ${ }^{10}$.

The aim of this study was to identify and describe prescription errors involving chemotherapeutic drugs and other medications in a centre for the preparation of injectable drugs (CPID) at a university hospital.

\section{Methods}

A retrospective descriptive study was carried out based on information from a center for the preparation of injectable drugs on prescription errors identified in the pharmaceutical validation stage before the preparation of the drug, in the period from 2016 to 2017. The hospital at the study site presents itself as a general, tertiary-level and university institution with 843 beds that serves a mean of 1,600 outpatients for cancer treatment and 1,600 hospitalizations/year in oncology units- adult and pediatric; also, the pharmacists at the center participate in clinical studies, mostly from phase 3 , which involve the handling of injectable drugs and infusion at the institution's research center - a mean of 700 prescriptions/year. Thus, the CPID is part of the Pharmacy Service, being the area responsible for handling and dispensing chemotherapy (parenteral and oral) and their adjuvants, and also for handling other injectable drugs (immunosuppressants, antibiotics, antifungals, total parenteral nutrition) in biological safety booths in properly classified areas and with microbiological controls (surface, environment, and manipulators).

At the hospital there are prescription and electronic medical records and dispensation of drugs by barcode; for prescription involving chemotherapy, in the electronic prescription system, the prescriber selects the desired protocol (cycle, medication), including weight and height data for calculating the dose in $\mathrm{mg} /$ $\mathrm{m}^{2}$ and administration guidelines, such as route (intravenous, oral, and intra-arterial, among others), drip and/or duration (in hours or $\mathrm{mL} / \mathrm{min}$ ), final volume, diluent (type of solution for diluting the medicine), and frequency (for example, once a day). The prescriptions for chemotherapy and other medications for manipulation are validated from 8 am to $10 \mathrm{pm}$ by pharmacists in the area, before preparation and/or dispensation; in this case, oral chemotherapy. All the processes are organized and recorded in order to maintain product and process traceability. The CPMI pharmacists make a technical assessment of the drugs that will be handled (dose, compatibility, concentration, route, stability); questions related to the prescription of drugs other than those mentioned in the oncology and hematology protocols such as analgesics, antihypertensives, sedatives, antiemetics, antibiotics, and antifungals, among others are evaluated by the clinical pharmacists of the service; the prescriptions with these drugs are validated in terms of dosage, route of administration, schedule, presentation/pharmaceutical presentation, and therapeutic indication, before dispensation. The hospital's distribution system consists of a unit dose for 24 hours and all the prescriptions are validated by pharmacists in their entirety.

Based on the records, the identified prescription errors were classified according to the guideline of the American Society of Health-System Pharmacists (1993), classifying them as follows: incorrect drug selection, underdose, overdose, pharmaceutical presentation, route of administration, dosage, therapeutic duplicity, diluent (lack of same or inadequate volume), drug incompatibility (pharmaceutical interaction), and inadequate treatment time, among others ${ }^{11}$. After identifying the error or non-conformity in the prescription, a pharmaceutical intervention was performed with the prescribing teams, for the most part, by telephone contact with the prescriber to justify the prescription item(s); being transferred to electronic medical records. The interventions were recorded and tabulated, as well as their results (adherence of the medical teams to the pharmaceutical interventions). The medications related to prescription errors and their frequency were identified.

The data were stored, processed, and analyzed using SPSS, version 18.0. The analysis was performed using descriptive statistics. The study was approved by the hospital's Research Ethics Committee (No. 16-0484).

\section{Results}

During the study period, a mean of 1,516 prescriptions per month were evaluated, with a mean of 4.5 items per prescription, and 562 prescription errors involving medications were identified, with approximately $2 \%$ more than one error in the same 
prescription being identified. The monthly mean value found was 23.4 errors. Table 1 presents the data regarding the validations and interventions in the prescriptions.

Table 1. General characteristics of the evaluated items.

\begin{tabular}{|c|c|c|c|c|}
\hline Items evaluated & 2016 & 2017 & $\begin{array}{l}\text { Monthly } \\
\text { mean }\end{array}$ & Mean \pm SD \\
\hline Validated prescriptions & 18,133 & 18,259 & $1,516.0$ & $18,196 \pm 89.1$ \\
\hline Outpatient prescriptions & 11,225 & 11,263 & 937.0 & $11,244 \pm 26.9$ \\
\hline Hospitalization prescriptions & 6,059 & 6,379 & 518.0 & $6,219 \pm 226.3$ \\
\hline $\begin{array}{l}\text { Prescriptions for clinical } \\
\text { studies }\end{array}$ & 849 & 617 & 61.1 & $733 \pm 164.0$ \\
\hline $\begin{array}{l}\text { Pharmaceutical interventions } \\
\text { in the prescriptions }\end{array}$ & 99 & 431 & 22.0 & $265 \pm 234.8$ \\
\hline Prescription errors identified & 99 & 460 & 23.4 & $279.5 \pm 255.3$ \\
\hline
\end{tabular}

In $94.3 \%$ of the prescriptions identified with errors ( $n=532$ ), pharmaceutical interventions were necessary for their correction, with a mean of 22 interventions per month. Of these, in $31.8 \%$ $(n=169)$ of the cases it was necessary to contact the teams and in $68.2 \%(n=363)$, it was not possible to contact the prescribers; however, the pharmaceutical intervention or conduct adopted was recorded in the electronic medical record. Of the pharmaceutical interventions performed, there was adherence in $99.6 \%$ of the cases.

In the period covered by the study, the rate of prescription errors involving drugs was $1.5 \%$ of the total prescriptions analyzed. The year 2017 was responsible for $82.4 \%$ of the prescription error records. The types of prescription errors are shown in Table 2.

Table 2. Classification of the prescription errors identified $(n=562)$.

\begin{tabular}{lc}
\hline Types of errors & $\mathbf{n}(\%)$ \\
\hline Lack of diluent associated with the medication & $315(56.0)$ \\
Diluent volume outside the maximum allowed & $127(22.6)$ \\
concentration & $24(4.3)$ \\
Therapeutic duplicity & $24(4.3)$ \\
Medication overdose & $20(3.6)$ \\
Medication underdose & $15(2.7)$ \\
Inadequate dosage & $12(2.1)$ \\
Route of administration & $9(1.6)$ \\
Incompatibility between drugs & $5(0.9)$ \\
Incorrect selection of the medication & $2(0.4)$ \\
Time of treatment & $1(0.2)$ \\
Pharmaceutical presentation & $8(1.4)$ \\
Others &
\end{tabular}

The most frequent prescription errors are related to the diluents associated with the preparation of the drugs, either in the absence of this information or in the prescription of volumes outside the concentration range required by the preparation of the drug with $56 \%$ and $22.6 \%$, respectively. Less frequent prescription errors and with a higher risk of causing harm to the patient were identified, such as therapeutic duplicity $(4.3 \% ; n=24)$, overdose $(4.3 \% ; n=24)$, underdose $(3.6 \% ; n=20)$, and inadequate dosage $(2.7 \% ; n=15)$.

The medications most frequently involved in the prescription errors are presented in Table 3.
Table 3. Medications involved in the prescription errors $(n=562)$.

\begin{tabular}{|c|c|c|}
\hline Medications & $\begin{array}{l}\text { Related } \\
\text { errors } \\
\text { n (\%) }\end{array}$ & Error description (\%) \\
\hline Cisplatin & $211(37.5)$ & Missing or adjusting the diluent (36.8) \\
\hline Etoposido & $79(14.1)$ & $\begin{array}{l}\text { Concentration adjustment (11.9) } \\
\text { Missing or adjusting the diluent (1.2) }\end{array}$ \\
\hline Carboplatin & $50(8.9)$ & $\begin{array}{l}\text { Concentration adjustment (6.9) } \\
\text { Missing or adjusting the diluent (1.2) }\end{array}$ \\
\hline Cyclophosphamide & $32(5.7)$ & Missing or adjusting the diluent (5.0) \\
\hline Oxaliplatin & $23(4.1)$ & $\begin{array}{l}\text { Concentration adjustment (1.8) } \\
\text { Incompatibility }(0.7)\end{array}$ \\
\hline Irinotecan & $18(3.2)$ & Missing or adjusting the diluent (3.0) \\
\hline Filgrastima & $16(2.8)$ & Duplicity (1.6) \\
\hline Ganciclovir & $14(2.5)$ & $\begin{array}{l}\text { Route of administration (0.9) } \\
\text { Overdose }(0.5)\end{array}$ \\
\hline Cytarabine & $12(2.1)$ & Missing or adjusting the diluent (1.4) \\
\hline Methotrexate & $12(2.1)$ & Missing or adjusting the diluent (0.7) \\
\hline Others & $2(0.4)$ & Contact with prescriber $(0.4)$ \\
\hline
\end{tabular}

\section{Discussion}

Errors with chemotherapy drugs occur frequently and have a high potential to cause serious harms to patients; it is estimated that chemotherapy drugs are responsible for $10 \%$ to $20 \%$ of the errors involving drugs ${ }^{7}$.

Although there are safety barriers such as electronic prescription and medical records and there is a bar code to control the processes of prescription, preparation, and dispensation, there is a need for the professionals' decision to select and apply the final conduct, allowing for the occurrence of errors or failures and, therefore, educating the prescribers is among the strategies for reducing prescription errors in hospitals ${ }^{12}$. An electronic medical record and prescription system has advantages such as eliminating writing problems or confusion with similar medication names, speed and control in the medication dispensation processes, reduction in the choice of incorrect medications, integration of medical records, exams and adverse events, ease in calculating doses, and identification of possible drug interactions ${ }^{7,13}$. A study identified that a computerized prescription system associated with the team's intervention reduced serious errors by 55\%, going from 10.7 adverse events per 1,000 patient-days to 4.86 adverse events ${ }^{14}$.

It is known that the majority of errors involving medications occur at the prescription (39\% to $56 \%$ of the cases) and administration (34\% to $38 \%$ of the cases) phases, with the stage involving dispensation responsible for $4 \%$ to $11 \%$ of the errors ${ }^{15}$. Data on the errors identified and reported by the professionals of the institution of this study, between 2010 and 2011, showed that $48.25 \%$ of the errors were related to prescriptions, being identified mostly by the nursing area; among the most common prescription errors identified were ambiguous care guidelines (for example: if necessary, according to medical advice), therapeutic duplicity, and problems with units of measurement (for example: milligrams, grams, milliliter, drops) ${ }^{16}$.

In a study carried out by Díaz-Carrasco et al (2007) it was identified that, out of a total of 135 errors involving chemotherapy and treatment adjuvants, $38.5 \%$ were related to dose and $21.5 \%$ to omission (mesna, premedication, acid folinic, antiemetics) ${ }^{17}$. In our study, the majority of the prescription errors, for being an 
area of handling and dispensation, were related to the lack or adjustment of diluents for the preparation of injectable drugs in prescriptions and, therefore, not being an error causing greater harm so it can be considered as an omission error ${ }^{18}$. This data also corroborates data found by Slama et al (2005), where most of the errors were related to the physicochemical properties of the drugs, affecting the final preparation of the medication ${ }^{19}$. The errors related to doses or dosages, although not the most frequent ones, can generate a sequence of failures, from the prescription of the medication by the physician or the dispensation by the pharmacy, to the administration in the patient by the nurse, which can trigger serious harms if not intercepted by the pharmacy or nursing professionals ${ }^{20}$. In this way, double checking at the time of prescription validation can be a way of ensuring greater safety for the process, verifying calculations of doses, dosage and frequency, protocol cycle, and questions related to preparations (volume, preparation stability, concentration maximum, access route) ${ }^{21}$.

A review by Schwappach and Wernli (2010) identified that, among the chemotherapeutic drugs most involved in errors are methotrexate $(15 \%)$, cytarabine $(12 \%)$, and etoposide $(8 \%)^{7}$. In our study, among the drugs most involved in prescription errors, we found the following: cisplatin (37.5\%), because it is a chemotherapy drug that, depending on the prescribed dose, generates no need to adjust the diluent volume due to the adequacy of the medication volume ( $1 \mathrm{mg} / \mathrm{mL}$ presentation) with the diluent for the final volume; etoposide (14.1\%), as it has a preparation protocol at the maximum concentration of $0.4 \mathrm{mg} /$ $\mathrm{mL}$; carboplatin (8.9\%), where the diluent adjustment follows the maximum concentration of $6 \mathrm{mg} / \mathrm{mL}$ in glucose $5 \%$ and 2 $\mathrm{mg} / \mathrm{mL}$ in sodium chloride $0.9 \%$, requiring adjustment of diluent volume and, occasionally, its exchange; cyclophosphamide (5.7\%), presenting lack of diluent information or requiring adjustment of its volume; and oxaliplatin (5.7\%) incompatible with $0.9 \%$ sodium chloride. Among the drugs that showed less frequent prescription errors are filgrastime, with a double prescription, and ganciclovir, with the wrong prescribed dose (total dose 2 or 3 times a day), and double prescription with different administration routes for the same patient (oral and intravenous routes).

In the study, there was an increase in the identification of prescription errors and pharmaceutical interventions in 2014. Such increases were due to changes in the conduct of the prescription analysis by the pharmacists and to the adoption of institutional strategies to reduce adverse events and promote greater patient safety. Among them, the institutionalization of the pharmaceutical validation process for prescribing is found in $100 \%$ of the cases ${ }^{22}$. According to Oliboni and Camargo (2009), the prescription validation process consists of verification stages in which the participation of the pharmacist shows great importance, from the receipt and evaluation of the prescription to the pre-manipulation of the drugs with protocol final product checking, enhancing the role of the multidisciplinary team in ensuring the safety of patients undergoing cancer treatment ${ }^{23}$.

As previously mentioned, the professionals' vigilant attitude regarding errors in the processes involving medications resulted in a greater record of prescription errors, which can be observed in the period of 2014. Dalmolin et al (2013) evaluated spontaneous notifications of errors in the same institution of the study for a period of 2 years, and an increase of $5.2 \%$ was observed in the notifications of errors that reached the patients, but without causing harm, suggesting that the professionals have been adopting the practice of reporting situations of errors or quasifailures within the patient safety culture and in the processes ${ }^{16}$.
As future perspectives, new approaches can be implemented in order to improve safety in the prescription processes before the preparation and dispensation of the medications to the patients, and many institutions have been looking for strategies to improve and prevent medication errors ${ }^{24,25}$. Among the suggested conducts are maintaining double pharmaceutical check of the prescription, improvements in the computerized prescription system based on the information selected by the prescriber based on the protocols, improving the product labeling information, such as automatic selection of the infusion time, preparation stability, medications and care for conservation and administration.

Regarding the diluents, the system can allow for automatic selection of the diluent compatible with the medication; however, there is a need to adjust the final concentration of the preparation (volume of the solution) and to check the presentation of the drug (different for each manufacturer). The pharmaceutical interventions in medical prescriptions must be duly registered in medical records, guaranteeing the safety of the processes and collaborating to qualify the care provided to patients undergoing cancer treatment.

\section{Conclusion}

Data on the identification of prescription errors are well described in the literature, but the process involving the validation, identification, and description of such errors in a center for hanlding injectable drugs, which receives a large number of prescriptions per day for preparation and dispensation, shows the fragility of the prescribing system, even though it is computerized, and the importance of barriers or organized processes to avoid errors. This because, in addition to avoiding the error in preparation and, especially, preventing it from reaching the patient, issues related to the cost of the prepared drug and the exposure time of the professional in handling must also be takeninto account. In this way, we can say that this study met the objective of evaluating the routine of a center for the preparation of injectable drugs in the identification and description of prescription errors involving chemotherapy, as well as the weaknesses in the process. Improvements in the prescription process such as updating the drug registry with maximum concentration dependent on minimum diluent volume, and electronic barriers that indicate therapeutic duplicity and overdose, are being implemented in the testing phase.

\section{Funding sources}

FIPE-Hospital de Clínicas de Porto Alegre.

\section{Collaborators}

LS and SN wrote and evaluated the study design, TJ, LS, GG collected and analyzed the data, CW performed statistical analysis. LS, GG and CW wrote and evaluated the article. TJ conducted a final review of the article.

\section{Conflicts of interest statement}

The authors declare that there are no conflicts of interest regarding this article. 


\section{References}

1. Dhawan I, Tewari A, Sehgal S, et al. Medication errors in anesthesia: unacceptable or unavoidable? Braz J Anesthesiol. 2017; 67(2): 184-92

2. Aronson JK. Medication errors: definitions and classification. $\mathrm{Br}$ J Clin Pharmacol. 2009; 67(6): 599-604.

3. World Health Organization (WHO). IBEAS: a pioneer study on patient safety in Latin America - Towards safer hospital care. Geneva: World Health Organization Study, WHO Press; 2011 Available in: www.who.int/patientsafety/research/ibeas _ report_en.pdf. Accessed on October 15, 2016.

4. Hepler CD. Improving the Quality of Medications Use: The Case for Medication Management Systems. In: Pharmaceutical Care Network for Europe Third International Working Conference on Pharmaceutical Care Research. Denmark; 2003. Available in: http://file.cop.ufl.edu/pop/hepler/apha/ MedUseSystemsPaper-2.pdf. Accessed on Aug 4, 2016.

5. Gandhi TK, Bartel SB, Shulman LN, et al. Medication safety in the ambulatory chemotherapy setting. Cancer. 2005; 104(11): 2477-83.

6. Knez L, Laaksonen R, Duggan C. Evaluation of clinical interventions made by pharmacists in chemotherapy preparation. Radiol Oncol. 2010; 44(4): 249-56.

7. Schwappach DLB, Wernli M. Medication errors in chemotherapy: incidence, types and involvement of patients in prevention. A review of the literature. Eur J Cancer Care. 2010; 19 (3): 285-92.

8. Neville H, Broadfield L, Harding C. Chemotherapy Order Entry by a Clinical Support Pharmacy Technician in an Outpatient Medical Day Unit. Can J Hosp Pharm. 2016; 69(3): 202-8.

9. Fernandes RF, Marsola APZC, Marin Arado GM, et al. Intervenções farmacêuticas em prescrições ambulatoriais de medicamentos antineoplásicos no Hospital das Clínicas de Ribeirão Preto - USP. Qualidade HC. 2012; 3: 105-9.

10. Souza M, Santos H, Santos M, et al. Atuação do farmacêutico hospitalar na oncologia. Boletim Geum. 2016; 7(1): 54-63.

11. American society of Health-System Pharmacists (ASHP). Guideline on preventing medication errors in hospitals. Am J Hosp Pharm. 1993; 50: 305-14.

12. Bos JM, Van den Bemt PM, Smet PA, et al. The effect of prescriber education on medication related patient harm in the hospital: a systematic review. Br J Clin Pharmacol. 2017; 83(5): 953-61.

13. Koppel R, Metlay JP, Cohen A, et al. Role of computerized physician order entry systems in facilitating medications errors. JAMA. 2005; 293(10): 1197-203.

14. Bates DW, Leape LL, Cullen DJ, et al. Effect of computerized physician order entry and a team intervention on prevention of serious medication errors. JAMA. 1998; 280 (15): 1311-6.

15. Bates DW, Cullen DJ, Laird N, et al. Incidence of Adverse Drugs Events and Potential Adverse Drugs Events - Implications for prevention. JAMA. 1995; 274(1): 29-34.

16. Dalmolin GRS, Rotta ET, Goldin JR. Medication errors: Classification of seriousness, type, and of medications involved in the reports from a University Teaching Hospital. Braz J Pharm Sci. 2013; 49(4): 793 - 802.

17. Díaz-Carrasco MS, Pareja A, Yachachi A, et al. Prescription errors in chemotherapy. Farm Hosp. 2007; 31 (3): 161-4.

18. Mathaiyan J, Jain T, Dubashi B, et al.Prescription errors in cancer chemotherapy: Omissions supersede potentially harmful errors. J Pharmacol Pharmacother. 2015; 6(2): 83-7.

19. Slama C, Jerome J, Jacquot C, Bonan B. Prescription errors with cytotoxic drugs and the inadequacy of existing classifications. Pharm World Sci. 2005; 27(4):339-43.

20. Ford CD, Killebrew J, Fugitt P, Jacobsen J, et al. Study of Medication Errors on a Community Hospital Oncology Ward. J Oncol Pract. 2006; 2 (4): 149-54.

21. Mangino PD. Role of the pharmacist in reducing medication errors. J Surg Oncol. 2004; 88 (3): 189-94.

22. Fabiá AS, Rodrigo EC, Marí AA, Cubells DA, et al. Pharmaceutical validation as a process of improving the quality of antineoplastic treatment. J Oncol Pharm Pract. 2005; 11(2):45-50.

23. Oliboni LS, Camargo AL. Validação da prescrição oncológica: o papel do farmacêutico na prevenção de erros de medicação. HCPA; 2009; 29(2): 147-52.

24. Instituto para el Uso Seguro de los Medicamentos (ISMP). Recomendaciones para La prevención de errores de medicación. Salamanca: ISMP-España Boletín no 35; 2012. Available in: http://www.ismp-espana.org/ficheros/ Boletin\%2035-\%200ctubre\%202012.pdf. Accessed on 17 Nov 2016.

25. Institute for Safe Medication Practices ISMP). Targeted Medication Safety Best Practices for Hospitals. ISMPEUA; 2018-2019. Available in:http://www.ismp.org/tools/ bestpractices/TMSBP-for-Hospitalsv2.pdf. Accessed on February 9, 2016. 